\title{
RECONOCIMIENTO Y SENSIBILIZACIÓN DE LA IDENTIDAD CULTURAL DE LA UNIDAD EDUCATIVA DEL MILENIO INTERCULTURAL BILINGÜE CHIBULEO
}

\author{
Lcda. Betty Gutiérrez ${ }^{1}$, Licda. Lima Quinatoa ${ }^{2}$, Licda. Jeaneth Villacis ${ }^{3}$, Licda. Anita Cañar ${ }^{4}$,Psc. Verónica \\ Elizabeth Olalla ${ }^{5}$
}

\begin{abstract}
Cultural identity is formed by a set of values, traditions, and beliefs of the different and different modes of behavior presented by human beings in the course and development of life, these are elements of paramount importance within a social group the same which serve to properly act and consolidate the sense of belonging and self-esteem that forms part of the diversity and interests of the developing human being, also corresponds to an important part in the aspect of individual identities, groups social are elements that allow us to identify, characterize and show us that we have several aspects in common and that we differentiate ourselves from others in places or cultures according to the space to which each one belongs, knowing and understanding that culture is what identifies the human being within place where one lives or has lived.
\end{abstract}

\section{Keywords}

Recognition, Sensitization Identity, Cultural Educational Unit, Bilingual Intercultural

\section{CÓDIGO UNESCO: Educación 531204}

\section{RESUMEN}

La Identidad cultural está formada por un conjunto de valores, tradiciones, y creencias de los diferentes y distintos modos de comportamiento que presentan los seres humanos en el transcurso y desarrollo de la vida, estos son elementos de suma importancia dentro de un grupo social los mismos que sirven para actuar adecuadamente y que se consolide el sentimiento de pertenencia y autoestima que hace parte a la diversidad y de los intereses del ser humano en vías de desarrollo, también corresponde a una parte importante en cuanto al aspecto de las identidades individuales, los grupos sociales son elementos que permiten identificarnos, caracterizarnos y mostrarnos qué tenemos varios aspectos en común y qué nos diferenciamos de otros en lugares o culturas según el espacio al que cada uno

${ }^{1}$ Unidad Educativa Tamboloma; Ambato, Ecuador; betydelpilar1@hotmail.com

${ }^{2}$ Unidad Educativa Gonzales Suarez; Ambato, Ecuador; mercedesilove_1972@hotmail.com

${ }^{3}$ Unidad Educativa Alonzo Castillo; Ambato, Ecuador; janethvillacis@yahoo.es

${ }^{4}$ Unidad Educativa Francisco Flor; Ambato, Ecuador; glory_anita11@hotmail.com

${ }^{5}$ Universidad Tecnológica Indoamericana; Ambato, Ecuador; elizabeth_olalla@hotmail.com 
pertenece, sabiendo y entendiendo que la cultura es lo que identifica al ser humano dentro del lugar en donde se vive o ha vivido.

\section{Palabras clave:}

Reconocimiento, Identidad Sensibilizadora, Unidad Educativa Cultural, Intercultural Bilingüe

\section{INTRODUCCIÓN}

El reconocimiento y sensibilización de la identidad cultural de la Unidad Educativa del Milenio Intercultural Bilingüe Chibuleo se presenta como una necesidad a ser abordada en el campo específico de la consolidación y conservación de las raíces culturales que presenta cada pueblo indígena, y tiene como objetivo principal el promover e impulsar el reconocimiento de la identidad cultural para el fortalecimiento de la autoestima mediante la sensibilización y recuperación de los valores ancestrales, teniendo en cuenta que el rescate de esta identidad no es solo de beneficio personal sino que conlleva una gran cantidad de seguidores a niveles comunitarios, los mismos que por diversos motivos personales, familiares ancestrales o la misma adopción de características y culturas de fuera son aprendidas, aceptadas y asimiladas por las personas que se desarrollan y desenvuelven en el medio en el que vivimos, haciendo que esta adopción de identidad falsa provoque un deterioro muy grande en la constitución de las culturas establecida por sus ancestros.

La Identidad cultural es la respuesta a la pregunta ¿quién soy yo?, esta respuesta está acompañada del autoconocimiento y del autoconcepto como parte de un autoestima bueno que cada uno de nosotros asocia a la identidad personal más fuerte cuando la persona sabe quién es, se ama y sabe lo que quiere y así se convierte en una persona con un sano orgullo por sí mismo, la alta autoestima provoca que sus pensamiento se puedan lograr y convertirse en objetivos de vida y se puedan realizarlos a corto mediano y largo plazo y que los beneficios adquiridos de estos sean lo más productivos para ello y puedan reflejar su propia identidad y que esta autoestima sea la base de la superación personal, familiar y académica.

La cultura es todo aquello creado por el hombre producto del momento histórico, social y económico en el que se vive, la cultura debería ser el reflejo principal y más importante de nuestra sociedad ya que nos resulta ilógico pensar que sea exactamente igual a aquella que vemos en los diferentes medios de comunicación cada una de estas presenta sus características propias e individuales. La cultura ecuatoriana debería ser el fruto de nuestra historia y nuestro presente, con nuestras necesidades y particulares características así como es de objeto el estudio mismo del Pueblo Chibuleo en donde se requiere la consolidación más sustentada y fortalecida por las personas que son parte de ella y en donde se está trabajando para poder despertar mejores expectativas de vida y de superación gracias a lo que se ha consolidado con el paso del tiempo en cuanto a estudios, vivienda y demás aspectos básicos y primordiales para su desarrollo. 
La contribución del proyecto de Identidad Cultural juegan un papel muy importante en la transformación de los pueblos y en la transformación y modificación de los pensamientos que poseen las personas del pueblo indígena, este proyecto va más allá de arraigar una cultura de por vida lo que se busca es que el pueblo de Chibuleo vea la necesidad de mantener un autoestima elevado y que por medio de ello busque su superación personal y pueda crecer en todo sentido pero dando más énfasis al desarrollo académico que hoy en día está dotado de muchos beneficios y de tecnología de punta en donde se puede realizar estudios más profundos para llegar a ser como sus más altas autoridades y como las personas más reconocidas dentro del pueblo indígena.

\section{DESCRIPCIÓN DETALLADA DEL PROYECTO}

La identidad cultural se manifiesta principalmente a través del idioma, las tradiciones y costumbres, los valores generados en cada pueblo y las aspiraciones de cada habitante, la composición étnica y específica que ellos han generado.

Es notorio y de gran importancia los niveles de educación superior que debe alcanzar cada individuo dentro de la comunidad indígena, es necesario que se encuentre en sus posibilidades para acceder a un título profesional que pueda proveerle el sustento de vida adecuado a sus necesidades

Existen indicios de que a lo largo de la última década, en diferentes regiones del planeta, se ha incrementado el número de personas que cursan la educación terciaria o que egresan de ésta. "Todos los países de Latinoamérica han experimentado un crecimiento importante de la población con estudios universitarios en el período entre 1998 y 2006" (Nieto y Ramos, 2010).

El poseer un nivel académico adecuado hoy en día es de gran importancia y valor para cada uno de los individuos que se encuentran en desarrollo académico con expectativas de vida altas y enfocadas a un nivel de superación adecuado a sus aspiraciones, los cuales les proporcionara de las herramientas adecuadas para un desempeño profesional lleno de resultados positivos para cada individuo en donde su autoestima se elevara para poder sustentar su trabajo.

Hofheinz (2009) destaca que, entre 1995 y 2007, en los países de la Latinoamérica el volumen de estudiantes que se matricularon en una carrera universitaria o de educación superior aumentó en un 100\% (cfr.Landoni, 2007; Gerald y Hussar, 1997; National Center for Education Statistics, s.f.; Agarwal, 2008 y UNESCO, 2010).

Hermida (2011) menciona que, la identidad tiene diversos tipos. Una está dada por los genes -como el sexo que deviene en género por la determinación social- otra por el nacimiento - el sitio o la familia que da la nacionalidad- otra por la vida misma -la 
generacional-. Estas son impuestas, otras son opcionales: la religión, la profesión, el equipo de fútbol predilecto. La identidad cultural está dada desde el nacimiento por la familia, el grupo étnico y socio económico, así como la nacionalidad, a la que se pertenece.

Lo que nos da la pauta principal para saber que la identidad cultural está arraigada a nuestros ancestros y que por ende esta consolidación de identidades debe ser conservada y preservada en las nuevas generaciones para que perdure en el tiempo y se puedan seguir conservando los logros que se presentan en la actualidad.

Es necesario considerar con especial interés a los diferentes estudios e investigaciones efectuadas sobre las identidades culturales debido a que son parte principal de la formación de los pueblos y de las manifestaciones culturales propiamente dichas, que han existido a lo largo de la historia de la humanidad, siendo estas de gran apogeo por diferentes personalidades que hoy en día sobresalen en beneficio de la sociedad y de la cultura indígena tratando de arraigar y fomentar más su identidad cultural.

Se establece los siguientes objetivos generales y específicos. Promover el reconocimiento de la identidad cultural para el fortalecimiento de la autoestima mediante la sensibilización y recuperación de los valores ancestrales.

- Desarrollar nuevos ejercicios de gimnasia cerebral para facilitar la activación de conocimientos para el aprendizaje de la matemática para niños de Cuarto Año.

- Diseñar una Guía Didáctica con la descripción de los ejercicios de gimnasia cerebral y Reconocer los valores culturales para el rescate de las raíces ancestrales.

- Respetar las ideas y creencias de las distintas culturas indígenas

- Fomentar una autoestima elevada para conocer el interés del pueblo indígena mediante el testimonio de los líderes del pueblo Chibuleo

\section{FUNDAMENTACIÓN CIENTÍFICA TÉCNICA}

Olga, Lucia Molano( 2007), menciona que "El concepto de identidad cultural se comprende a través de las definiciones de cultura y de su evolución en el tiempo, El tema cultural es tratado desde varios aspectos: el económico, donde la cultura se vincula al mercado y al consumo y se manifiesta en las llamadas industrias culturales (empresas editoras, casas de música, televisión, cine, etc.); el humano, donde la cultura juega un papel de cohesión social, de autoestima, creatividad, memoria histórica, etc.; el patrimonial, en el cual se encuentran las actividades y políticas públicas orientadas a la conservación, restauración, puesta en valor, uso social de los bienes patrimoniales".

Los modos de comportamiento funcionan como elementos principales y primordiales dentro de un grupo social, comunidad, pueblo o cualquier espacio que determine un cierto 
límite de población, la identidad cultural está formada por varios aspectos arraigados al lugar de procedencia de los seres humanos, en un mundo lleno de tecnología, libertad, formas de vivir, diferente religiones se vuelve cada vez más complicado este tema de la conservación cultural ya que las nuevas generaciones se están formando con este nuevo estilo de vida en donde muchas veces la presión social lleva a que se desvincules de la parte ancestral y opten por nuevas formas de vivir, en cierta manera es de valor pero cuando el individuo se aleja totalmente de lo que por nacimiento este está siendo enfatizado por una gran falta de pertinencia y es en donde su autoestima tiende a desmejorar y a verse influenciado en tal ámbito que se dejan llevar por situaciones no adecuadas que les provocan daños a niveles personales, sociales y familiares.

Gabriela, Orduna Allegrini (2003), menciona que "Se puede afirmar que el desarrollo local es un cambio social innovador que implica directamente a todos los recursos comunitarios, pero de una forma especial a los recursos humanos, los responsables de impulsar y materializar el resto de acciones innovadoras. En atención a esos recursos humanos, a la comunidad, el desarrollo lleva consigo siempre una acción educativa encaminada a que las personas protagonistas de la innovación estén informadas, capacitadas y motivadas para emprenderla".

El auto definirse cuando se habla de identidad cultural implica un continuo contacto de socialización y apreciación de la misma persona en el contexto o medio de desarrollo en el que se encuentra, las relaciones o necesidades entre la comunidad no pueden ser iguales debido a que cada persona tiene diferentes interés de superación personal con diferentes objetivos de vida los cuales los desarrolla a medida de sus posibilidades y en como este se siente a gusto con la sociedad y sobre todo en medida de como aumenta su autoestima para tomar decisiones que le harán crecer en cualquier ámbito ya sea personal, familiar, social y sobre todo en el aspecto de la superación académica.

El hecho mismo de que dentro de una cultura o práctica cultural exista la conciencia de una identidad común, implica que también hay un impulso hacia la preservación de esta identidad, hacia la auto preservación de la cultura. Si la identidad es construida en oposición a los extraños, las intrusiones de otras culturas implican la pérdida de autonomía y por lo tanto la pérdida de identidad, lo que es más complicado volver a reconstruir, ya que por los diferente criterios que tiene las personas acerca de comunidad e identidad cultural muchas veces están arraigados a sus ancestros o como hoy en día se puede reflejar en el medio en que nos desarrollamos que esta identidad y autoestima se la está perdiendo y es más vulnerable a ser sujeto de modificación y adopción de otras identidades y formas de vivir.

Dentro de las principales características que se deben cumplir en cuanto a la conservación de identidad cultural y a la capacidad de elevar el autoestima se puede observar una mejora en la calidad de vida de los pueblos, la capacidad de establecer programas de educación en 
comunidades donde la mayoría de la población requiere de estos conocimientos adecuadamente planificados y establecidos para satisfacer las necesidades más importantes y relevantes de los sujetos con una consolidación arraigada a sus culturas ancestrales.

Gabriela, Orduna Allegrini (2003), menciona que "Toda experiencia de desarrollo comunitario, así como las acciones educativas que llevan parejas, se despliegan en un territorio, en un momento delimitado y con una duración determinada. En lugar de una acción aislada que pretende solucionar un problema concreto, se trata de una cadena de actuaciones que aspiran a elevar la calidad de vida de la comunidad y que forman un proceso continuo de experiencias entrelazadas extendidas sin interrupción en el tiempo."

Organizar campañas en el ámbito cultural y valores de autoestima serían muy beneficiosos para ampliar el conocimiento de la sociedad sobre el valor de la cultura ancestral, y por medio de la educación promover sobre los Derechos Humanos, para concientizar a la comunidad sobre temas de actualidad, y apoyar el desarrollo de programas culturales, educativos $\mathrm{y}$ de investigación sobre las diferentes comunidades fomentando el conocimiento de diversas culturas y tradiciones nacionales y lograr el reconocimiento y la protección efectiva de los derechos individuales y colectivos del pueblo indígena.

Louise-Anne Beauregard, Richard Bouffard, Germain Duclos (2005) menciona que, "La autoestima constituye uno de los principales factores del desarrollo humano; que es el fundamento de la educación. La obra educativa consiste esencialmente en acompañar o guiar a los niños en su vida afectiva, social y moral. El educador debe favorecer siempre la autoestima, porque constituye la condición para poder compartir y socializar, y para vivir relaciones armoniosas con el entorno".

Reynald Legendere (2005) define "La autoestima como el valor que un individuo se concede globalmente; hace alusión a la confianza fundamental que el ser humano deposita en sus propios recursos, en su eficacia y en sus capacidades".

Es por eso que los talleres de este proyecto están basados en elevar el autoestima ya que por medio de los mismos se busca elevar los diferentes criterios que se tiene acerca de uno mismo y de cómo este pensamiento puede cambiar y mejorar para beneficio personal y a favor de la sociedad.

Jean R. Feldman (2000) menciona. "La autoestima incluye todos los sentimientos y creencias que tenemos acerca de nosotros mismos y afecta a todo lo que hacemos en la vida. Es compleja, cambiante y está intrincadamente entretejida con nuestro ser. Un aspecto de la autoestima es la aceptación propia: conocerse a sí mismo, tener una idea realista de lo que somos y estar conformes con ello. Las experiencias de éxito influyen en la propia eficacia y en la creencia de que uno tiene la capacidad de enfrentarse con la vida. Otro componente es el autorrespeto, porque sólo cuando uno se respeta a sí mismo puede 
acercarse a otros con respeto y aceptación. La confianza en nosotros mismos nos prepara para abordar cosas nuevas".

La necesidad de elevar el autoestima y mejorar los niveles de vida tiene como finalidad que los integrantes de la comunidad de Chibuleo superen sus expectativas de vida y puedan superar sus conocimientos por medio de una educación más formal y complementaria a sus capacidades de superación y que aprendan a ver la vida de una manera diferente haciendo uso de todos los recursos que se les presenta a su alrededor los mismos que hoy en día tienen todos los beneficios para lograr grandes superaciones.

Christophe André, François Lelord (2000) menciona. "Tener confianza en uno mismo, es estar seguro de sí, estar contento de sí, los términos y las expresiones empleadas en el lenguaje corriente para designar la autoestima son incontables. De hecho, cada uno de ellos se refiere a uno de sus múltiples aspectos. En realidad, la autoestima consta de tres ingredientes: la confianza en sí mismo, la visión de sí mismo, el amor a sí mismo, la buena dosificación de cada uno de esos tres componentes es indispensables para obtener una autoestima armoniosa".

Unidades Educativas del Milenio "Guardianas de la Lengua" preservan y revitalizan las lenguas ancestrales

El objetivo de trabajar con esta Unidad Educativa del Milenio radica en la identificación de la perdida de la identidad cultural y la baja autoestima que se genera debido a la influencia del medio en que los habitantes del pueblo Chibuleo se desenvuelven es por eso que se vio la necesidad de mencionar una mini reseña historia de la creación de la Unidad Educativa amparada en el Ministerio de Educación que a continuación se detalla: Con el objetivo de continuar con el fortalecimiento de la Educación Intercultural Bilingüe, esta mañana, el viceministro de Educación, Freddy Peñafiel, inauguró la política "Guardianas de la Lengua", que busca crear y fortalecer instituciones educativas emblemáticas en las que se aplicará al 100\% las innovaciones del Modelo del Sistema de Educación Intercultural Bilingüe (Moseib), además de preservar y revitalizar las lenguas ancestrales de las nacionalidades y pueblos indígenas del Ecuador.

La presentación de esta política fue realizada en la Unidad Educativa del Milenio (UEM) Intercultural Bilingüe "Chibuleo", que será la encargada de preservar la lengua, los conocimientos y los saberes ancestrales de la nacionalidad Kichwa, del pueblo Chibuleo. Esta institución del milenio es la primera "Guardiana de la Lengua", de las 14 que existirán para cada una de las nacionalidades del país hasta el 2017.

En su discurso, el viceministro de Educación resaltó la importancia de esta política para la transformación del país. "Con la construcción de las Unidades Educativas del Milenio «Guardianas de la Lengua» no solo estamos cambiando la educación del país; estamos 
cambiando nuestras vidas, porque estamos seguros que de estas instituciones saldrán los próximos líderes de este país", señaló Peñafiel.

Cada una de las Unidades Educativas del Milenio "Guardianas de la Lengua" estará ubicada en sectores donde existe mayor concentración poblacional de cada nacionalidad y tendrá la misión de fortalecer, conservar, promover la lengua ancestral, así como de custodiar las cosmovisiones de las nacionalidades y establecer un diálogo constante entre los saberes ancestrales y el conocimiento occidental.

El viceministro de Educación, a más de inaugurar esta política educativa, anunció que la UEM Intercultural Bilingüe "Chibuleo" es candidata para ofertar el Bachillerato Internacional, "para que los estudiantes que se gradúen de esta institución no solo tengan oportunidades de acceso a universidades nacionales, sino además puedan estudiar en las mejores universidades del mundo".

Por su parte, Selena Andagana, estudiante de $3 .^{\circ}$ curso de Bachillerato, habló en representación de sus compañeros. Selena recordó la historia de su pueblo al no poder contar con educación de calidad. "Hace mucho tiempo, los mestizos pensaban que nosotros, los indígenas, nacimos para ser jornaleros y trabajar solo en la tierra. Pero no solo pensaban eso los mestizos, sino nosotros mismos, los indígenas. Ahora, gracias la Revolución Ciudadana, esta realidad es diferente. Yo tengo la posibilidad de graduarme para luego estudiar en la universidad y ser doctora", manifestó la estudiante.

En cuanto a la Unidad Educativa del Milenio Intercultural Bilingüe "Chibuleo", esta cuenta con laboratorios de física, química, tecnología e idiomas; bloques de administración, de biblioteca y de aulas; sala de uso múltiple, bar, parqueaderos, baterías sanitarias, patio cívico, pizarras inteligentes, canchas deportivas y oferta educativa completa (desde Educación Inicial hasta Bachillerato).

Su construcción es de 2.500 metros, en un área de terreno de 3,5 hectáreas, cuyo costo fue de USD \$4'210.348,90.

Con esta breve reseña nos damos cuenta de la gran importancia que tiene el país al crear este tipo de Instituciones y por ende se entiende los beneficios que proporción el educarse y poder llegar a grandes niveles académicos con el objetivo de superación personal y a la final poder cumplir con los objetivo de vida planteados para cada sujeto en el contexto en donde se desarrollan.

\section{METODOLOGÍA}

La metodología de investigación está ligada a los objetivos de investigación.

Su utilizarán como fuentes de información primaria al pueblo de Chibuleo, estudiantes DE LA UNIDAD EDUCATIVA DEL MILENIO INTERCULTURAL BILINGÜE 
CHIBULEO en un número de 890 estudiantes distribuidos en toda la Unidad Educativa divididos en hombres y mujeres es importante mencionar que como variables se encuentran la edad, el género,, el nivel de formación actual . Todos los sujetos de investigación serán informados de los objetivos y de las características del estudio con el fin de evitar inconvenientes o herir susceptibilidades, se recalcarán las normas para la utilización de los talleres respetando la identidad, pensamiento y cultura de cada sujeto participante.

Los instrumentos será mediante la aplicación de talleres para elevar el autoestima y contribuir a la conservación de la identidad cultural, serán 5 talleres cada uno se identificara con su propio tema y el adecuado sistema de trabajo.

\begin{tabular}{|c|c|}
\hline ETAPAS & TAREAS \\
\hline $\begin{array}{l}\text { 1. Trabajo de recopilación teórica de los } \\
\text { contenidos para los talleres. }\end{array}$ & $\begin{array}{l}\text { - Trabajo de recopilación de fuentes de } \\
\text { información (trabajo autónomo, } \\
\text { selección de fuentes). } \\
\text { - Clasificación de temas para los talleres. }\end{array}$ \\
\hline $\begin{array}{l}\text { 2. Determinación de los tipos de talleres a } \\
\text { ser trabajados en la Unidad Educativa } \\
\text { para fomentar una autoestima elevada. }\end{array}$ & $\begin{array}{l}\text { - Organización de la información } \\
\text { - Verificar información } \\
\text { - } \quad \text { Aplicación de los talleres } \\
\text { - } \quad \text { Resultados de los talleres }\end{array}$ \\
\hline $\begin{array}{l}\text { 3. Describir qué factores están influyendo } \\
\text { en el desarrollo de la autoestima para } \\
\text { consolidarlo. }\end{array}$ & $\begin{array}{l}\text { Incorporar a los talleres el uso de los } \\
\text { recuerdos y valores ancestrales. }\end{array}$ \\
\hline $\begin{array}{l}\text { 4. Analizar cómo se puede mejorar el } \\
\text { autoestima de manera permanente y } \\
\text { que se convierta en un aprendizaje } \\
\text { significativo que les provee de las } \\
\text { herramientas adecuadas para un mejor } \\
\text { desarrollo y desenvolvimiento personal } \\
\text { en el medio en que cada sujeto se } \\
\text { encuentra. }\end{array}$ & $\begin{array}{l}\text { Difusión de los resultados de los } \\
\text { talleres a las máximas autoridades de la } \\
\text { Unidad Educativa. }\end{array}$ \\
\hline
\end{tabular}

Tabla $N^{\circ} 1$ : Etapas y tareas del proyecto

Elaboración: Grupo Investigador

La estadística será el resultado del análisis de todas los resultados obtenidos mediante los talleres de sensibilización y reconocimiento para un autoestima elevado y que por medio de ello se conserve la identidad cultural y que no se la deje de lado o supla por identidades ajenas a la propias y que servirán para la elaboración de las conclusiones y recomendaciones. 


\section{RESULTADOS ESPERADOS}

1. Memoria escrita de los tipos de recursos utilizados para el desarrollo adecuado de la autoestima mediante a la aplicación de los talleres preestablecidos, evidenciado en el informe final con perspectiva de elaboración de un artículo científico.

2. Memoria escrita de los temas de talleres presentes en los estudiantes de la Unidad Educativa Chibuleo en función de su formación, su edad, sexo y evidenciado en el informe final con perspectiva de elaboración de un artículo científico

3. Artículo científico resultante de las conclusiones del proyecto de RECONOCIMIENTO Y SENSIBILIZACIÓN DE LA IDENTIDAD CULTURAL DE LA UNIDAD EDUCATIVA DEL MILENIO INTERCULTURAL BILINGÜE

CHIBULEO a ser publicado en la revista de la Universidad con las descripción propia de los resultados obtenidos por medio de la aplicación de los talleres para elevar el autoestima.

\section{FINANCIAMIENTO}

Costo final de proyecto según se detalla en el cuadro

\begin{tabular}{lr}
\hline \multicolumn{1}{c}{ Recursos } & Costo total \\
\hline $\begin{array}{l}\text { Recursos humanos } \\
\text { Recursos físicos y } \\
\text { bibliográficos }\end{array}$ & 3840.00 \\
\hline Servicios especializados & 850.00 \\
\hline \multicolumn{1}{c}{ TOTAL } & 480.00 \\
\hline
\end{tabular}

Tabla $N^{\circ} 2$ : Recurso financieros

Elaboración: Grupo Investigador

Tiempo estimado de la ejecución del proyecto será de 12 mese acorde a los objetivos plateado

Objetivo Específico $\mathbf{N}^{\circ}$ 1. Reconocer e identificar los valores culturales para el rescate de las raíces ancestrales.

- Trabajo de recopilación de fuentes bibliográficas

- Clasificación de recursos para los talleres.

- Trabajo de recopilación de información acerca de los precursores en al ámbito indígena.

Objetivo Específico $\mathbf{N}^{\circ}$ 2. Respetar y valorar las ideas y creencias de las distintas culturas indígenas

- Verificar información

- Procesamiento de la información 
Objetivo Específico $\mathbf{N}^{\circ}$ 3. Promover y fomentar una autoestima elevada para conocer el interés del pueblo indígena mediante el testimonio de los líderes del pueblo Chibuleo

- Redacción del artículo-definición. Incorporar comentarios.

- Incorporar al artículo evidencia Multimedia. Uso de los recursos utilizados en los talleres de identidad cultural.

\section{SOSTENIBILIDAD}

Este tipo de investigaciones permite ver una forma abierta a la innovación y actualización de conocimientos con impacto en toda la comunidad indígena. La socialización en este tipo de talleres de identidad cultural en espacios de colaboración y construcción de conocimiento compartido y común, mediante actividades formativas permiten a los miembros de las comunidades ver más allá de lo que común mente tienen en su diario vivir y por ende harán que cada uno de ellos vayan modificando su pensamiento y comportamiento para mejorar sus objetivos de vida y que puedan sobresalir en el aspecto social, cultural, político, educativo como hoy en día se puede reflejar en el mismo medio en el que nos desarrollamos exaltando y haciendo relevancia a las personas que han logrado estos aspectos positivos en la comunidad indígena, esperando que los futuros profesionales presente esa necesidad de estar continuamente en formación y puedan hacer uso de lo que hoy en día se tiene para el progreso indígena, y por medio de ello se conserve y arraigue más la identidad cultural con un fortalecimiento propio y autóctono.

Estas experiencias de vida de los ancestro y precursores indígenas dejan muchas enseñanzas y no solo a la comunidad a la que cada uno pertenece sino en su totalidad porque se puede observar como los indígenas son ovacionados en países de fuera dejando en alto el nombre y costumbres de sus propias culturas, pero esto hay que fortalecerlo cada día desde los más pequeños para que en el transcurso de su desarrollo no pierdan la identidad cultural y que por presión del medio tiendan a cambiar su pensamiento y costumbres de vida, forjando en cada uno de ellos un autoestima bueno y elevando sus capacidades al máximo se podrán cumplir varias metas propuestas las mismas que serán en un futuro

Aprendizajes sociales manteniendo una educación sostenible y adecuada a las necesidades de cada uno de los individuos de los pueblos o comunidades.

Desde Postgrados de la Universidad Técnica de Ambato se propenderá a la utilización de estos mecanismos de enseñanza como una estrategia más de formación no solo en el ámbito profesional sino el elevar el autoestima de quien nos rodea contribuyendo a una sociedad más sustentable e inclusiva, ampliando la visión de servicio y superación para toda la sociedad. . 


\section{BENEFICIARIOS DEL PROYECTO}

\section{BENEFICIAROS DIRECTOS}

Los beneficiarios directos serán la comunidad de Chibuleo y en especial la UNIDAD EDUCATIVA DEL MILENIO INTERCULTURAL BILINGÜE CHIBULEO quienes serán beneficiados con este tipo de talleres de identidad cultural para elevará su autoestima y se puedan conocer a ellos mismos como parte importante de la sociedad, un numero inicial de 890 estudiantes. Así también los/as docentes autoridades de la institución ya que se utilizaría tecnología que posee la misma unidad educativa.

\section{BENEFICIARIOS INDIRECTOS}

Los beneficiarios indirectos será la misma comunidad ya que por medio de los estudiantes se puede llegar a ello de manera indirecta por medio de notificaciones para que puedan asistir a los diferentes talleres de identidad cultural propuestos por las investigadores del proyecto, el mismo que se lo realizara en espacios abiertos y con la coordinación adecuada y respectiva.

\section{FACILIDADES DE TRABAJO}

El presente proyecto está presentado como parte de los cambios que constantemente debemos tener como seres humanos en el transcurso y desarrollo de nuestra vida, y por la necesidad de elevar la autoestima y recuperar la identidad cultural que por varios motivos e intereses se van perdiendo.

Al ser una investigación Aplicada, cuenta con la colaboración y el apoyo de los representantes de otras instituciones lo que facilita su ejecución para lo cual se establecerán convenios de cooperación interinstitucional.

RECEIVED: SEPTEMBER, 2016

REVISED: DECEMBER, 2016

Bibliografia

1. UNESCO (2010) United Nations Educational 2010: The Current status of science around the world. Paris: UNESCO.

2. Nieto, S.; Ramos, R. (2010). "Sobreeducación, educación no formal y salarios: Evidencia para España”. Fundación de las Cajas de Ahorro Http://www.funcas.ceca.es/Publicaciones/InformacionArticulos/Publicaciones.asp? $\underline{I D=1650}$

3. Colmeiro, Jose, F. (2005), "Memoria Historica e identidad cultural de la posguerra a la postmodernidad", Barcelona.

4. Molano, Olga Lucía, (2007), "Identidad cultural un concepto que evoluciona.", Revista Opera, ISSN-e.

5. Orduna, Allegrini Olga, (2003)" Desarrollo local, educación e identidad cultural”, Departamento de Educación Universidad de Navarra. 
6. Louise-Anne Beauregard, Richard Bouffard, Germain Duclos (2005), “Autoestima: para quererse más y relacionarse mejor", Alemania

7. Reynald Legendere (2005), “Autoestima: para quererse más y relacionarse mejor", Alemania

8. Jean R. Feldman (2000), “Autoestima ¿cómo desarrollarla?: juegos, actividades, recursos, experiencias creativas”, Georgia

9. Christophe André, François Lelord (2000), "La Autoestima: gustarse a sí mismo para mejor vivir con los demás”, Estados Unidos.

10. Unidades Educativas del Milenio (2014), "Guardianas de la Lengua" preservan y revitalizan las lenguas ancestrales, "Ministerio de Educación Ecuador"

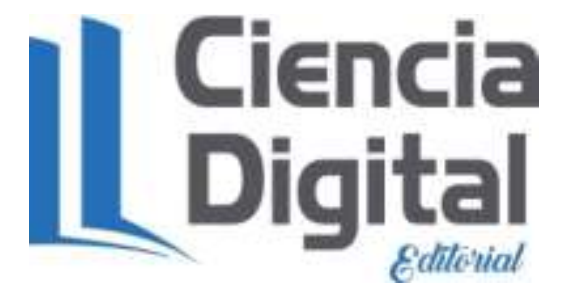

El artículo que se publica es de exclusiva responsabilidad de los autores y no necesariamente reflejan el pensamiento de la Revista Ciencia Digital.

El articulo queda en propiedad de la revista y, por tanto, su publicación parcial y/o total en otro medio tiene que ser autorizado por el director de la Revista Ciencia
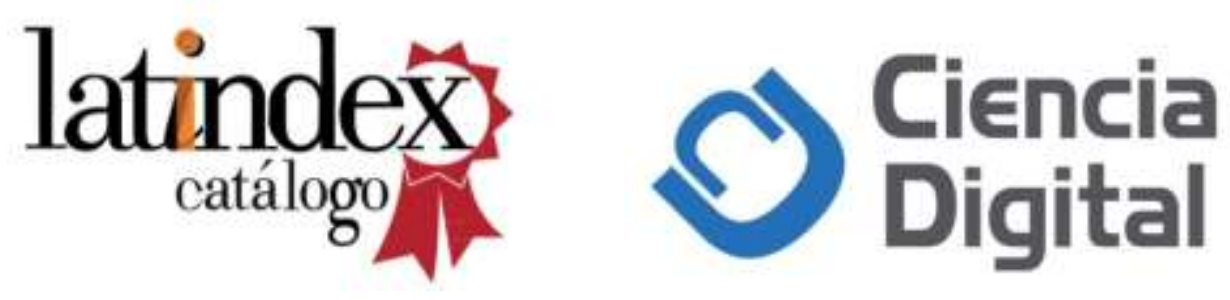